\title{
Uniportal video-assisted thoracoscopy is a safe approach in patients with empyema requiring surgery
}

\author{
Lars B. van Middendorp ${ }^{1,2}$, Stijn Franssen ${ }^{1}$, Sanne Gillissen ${ }^{1}$, Jos G. Maessen ${ }^{2}$, Karel W. E. Hulsewé ${ }^{1}$, \\ Yvonne L. J. Vissers ${ }^{1}$, Erik R. de Loos ${ }^{1}$ \\ ${ }^{1}$ Department of Surgery, Division of Thoracic Surgery, Zuyderland Medical Center, Heerlen, The Netherlands; ${ }^{2}$ Department of Cardiothoracic \\ Surgery, Maastricht UMC+, Maastricht, The Netherlands \\ Contributions: (I) Conception and design: LB van Middendorp, ER de Loos; (II) Administrative support: LB van Middendorp; (III) Provision of study \\ materials or patients: ER de Loos, YLJ Vissers, KWE Hulsewé; (IV) Collection and assembly of data: S Franssen, S Gillissen; (V) Data analysis and \\ interpretation: S Franssen, S Gillissen, LB van Middendorp, ER de Loos, JG Maessen; (VI) Manuscript writing: All authors; (VII) Final approval of \\ manuscript: All authors. \\ Correspondence to: Lars B. van Middendorp. P. Debyelaan 25, 6229 HX Maastricht, The Netherlands. Email: lars.middendorp@mumc.nl.
}

Background: Empyema is a well-known complication of pneumonia, with high morbidity and mortality rates. This warrants direct treatment either with antibiotics and chest tube drainage or surgery. With less invasive surgical approaches such as uniportal video-assisted thoracoscopic surgery (uVATS), surgical intervention gets a more prominent role early on in the treatment of empyema. The aim of this study was to compare uVATS with the complete VATS (cVATS) approach in empyema, with respect to postoperative complications, hospital length of stay and mortality.

Methods: All cases of empyema that were treated surgically in our hospital between 2006 and 2019 were included in a retrospective database. The preferential surgical approach changed from cVATS from 2006 to 2015, towards uVATS from 2016 and on, based on the experience of the surgical team. The database included pre- and postoperative data, as well as peropartive characteristics.

Results: One hundred and thirty-seven patients were treated with cVATS and 49 with uVATS. Apart from a slightly reduced kidney function in the uVATS group $\left(57.3 \pm 6.3\right.$ vs. $71.4 \pm 17.2 \mathrm{~mL} / \mathrm{min} / 1.73 \mathrm{~m}^{2}$, $\mathrm{P} \leq 0.001)$, there were no significant baseline differences in patient characteristics. The duration of uVATS was comparable to cVATS $(70 \pm 17$ vs. $56 \pm 23 \mathrm{~min}, \mathrm{P}=0.240)$, and with low per- and postoperative complications. The postoperative hospital stay was equal in both groups (19 \pm 13 vs. $20 \pm 15$ days, $\mathrm{P}=0.320)$. There were no statistically significant differences in postoperative complications or death.

Conclusions: Uniportal VATS is a feasible and safe technique for the use in patients with empyema requiring surgery. Even if decortication in stage III empyema is required this can be performed by uniportal VATS.

Keywords: Uniportal video-assisted thoracoscopy (uVATS); empyema; parapneumonic effusion; complete videoassisted thoracoscopy (cVATS)

Submitted Dec 10, 2019. Accepted for publication Jan 18, 2020.

doi: $10.21037 /$ jtd.2020.02.29

View this article at: http://dx.doi.org/10.21037/jtd.2020.02.29

\section{Introduction}

Empyema is a well-known complication of pneumonia prolonging hospital stay and often requires chest tube drainage or surgical evacuation of the pleural fluid (1). It is divided into three stages. Stage I is the exudative stage. Stage II is the fibrinopurulent stage, where the pleural fluid is thick or purulent and fibrin deposits develop over the pleural surface. This leads to the development of a pleural peel and loculation of fluid. Stage III is the well-organized 
and chronic phase with a thick peel of fibrin across the pleura (2). In stage I, chest tube drainage and treatment with systemic antibiotics is often sufficient to resolve the empyema. In this stage the lung is still compliant and can re-expand when the pleural fluid is evacuated. Surgical treatment is usually reserved for stage II and stage III, since the fibrin layer that is formed prevents the lung from re-expanding (3). Although no conclusive evidence is available of improved outcome regarding morbidity or mortality, the duration of chest drainage appears to be improved by surgical therapy in comparison to conservative treatment. Depending on the thickness of the pleural peel, decortication is required to free the trapped lung. Early surgical intervention can improve prognosis and shorten hospital stay (4). However non-surgical treatment of these ill patients is demanding, with high morbidity and mortality rates. Complication rates of surgical intervention vary around $20 \%$ and mortality rates as high as $13 \%$ have been reported $(5,6)$. Video-assisted thoracoscopic surgery (VATS) is currently the preferred approach for stage II empyema and is as effective as the classic open transthoracic approach $(7,8)$. Due to its less invasive nature, it has been suggested that VATS should be considered earlier in the treatment of empyema (9). Among the proposed benefits of a minimally invasive approach are reduced blood loss, decreased pain, shorter length of hospital stay and fewer complications $(10,11)$. Complete VATS (cVATS) has evolved from the standard 3 to 4 surgical ports, towards uniportal VATS (uVATS) in which a thoracoscopic procedure can be performed via a single small incision. In 2004 uVATS was introduced for a pulmonary wedge resection. Subsequently more complex procedures were performed and the technique gained in popularity (12). uVATS lobectomy already has similar postoperative outcomes as the multiportal approach and may be advantageous in postoperative pain control, mobility, length of chest tube duration and length of hospital stay $(13,14)$. In the current study we review our experience with uVATS treatment for empyema complicating pneumonia.

\section{Methods}

Data were retrospectively collected from the registry of our Thoracic Surgery Department. Approval was obtained from the local ethical committee. All cases of stage II and stage III empyema that were treated surgically between 2006 and 2019 were included. Patients with an uncomplicated parapneumonic effusion or stage I empyema, were treated conservatively and were excluded from analysis. Indication for surgery was deterioration of uncomplicated parapneumonic effusion into grade II or grade III empyema. This was based on either persistent pleural effusion despite chest tube drainage and antibiotic treatment, or signs of complicated effusion on CT or via transthoracic aspiration. Important CT findings included; signs of entrapped lung, atelectasis, loculated effusion, pleural thickening and persistent effusion despite chest tube drainage. Exclusion criteria were non-parapneumonic effusions, such as seen in Boerhaave syndrome or after a thoracic trauma, and empyemas treated with thoracotomy.

\section{Surgical technique}

There were no absolute contra-indications for cVATS or uVATS apart from general contra-indications for surgery. The preferential surgical approach for an empyema changed from cVATS in the period from 2006 to 2015 towards uVATS from 2016 and on, based on the experience of the surgical team. In all cases, the pleural cavity was thoroughly debrided and adhesions were removed in order to fully reexpand the lung. Indication for decortication (resection of the pleural peel) was a peropartive decision when trapped lung was found in the presence of a significant fibrous layer on the visceral pleura of one or more lobes.

\section{Data collection}

The following parameters were evaluated: (I) epidemiological data; age, sex, smoking habit, antibiotic treatment, chest tube and relevant co-morbidities such as diabetes mellitus, chronic obstructive pulmonary disease, cardiovascular diseases, malignancies and renal failure. (II) Postoperative characteristics; hospital stay, chest tube duration, any major postoperative complications including re-operations. (III) Peropartive characteristics including operating time, conversion rate to cVATS or thoracotomy and intra-operative complications. (IV) Laboratory results of the day prior to surgery, the first, third and fifth postoperative day.

\section{Statistical analysis}

Data were analysed using the statistical software package SPSS (version 25.0; SPSS Inc., Chicago, IL, USA). Descriptive statistical analysis is expressed as frequency, mean and standard deviation. Categorical variables were 
Table 1 Baseline characteristics

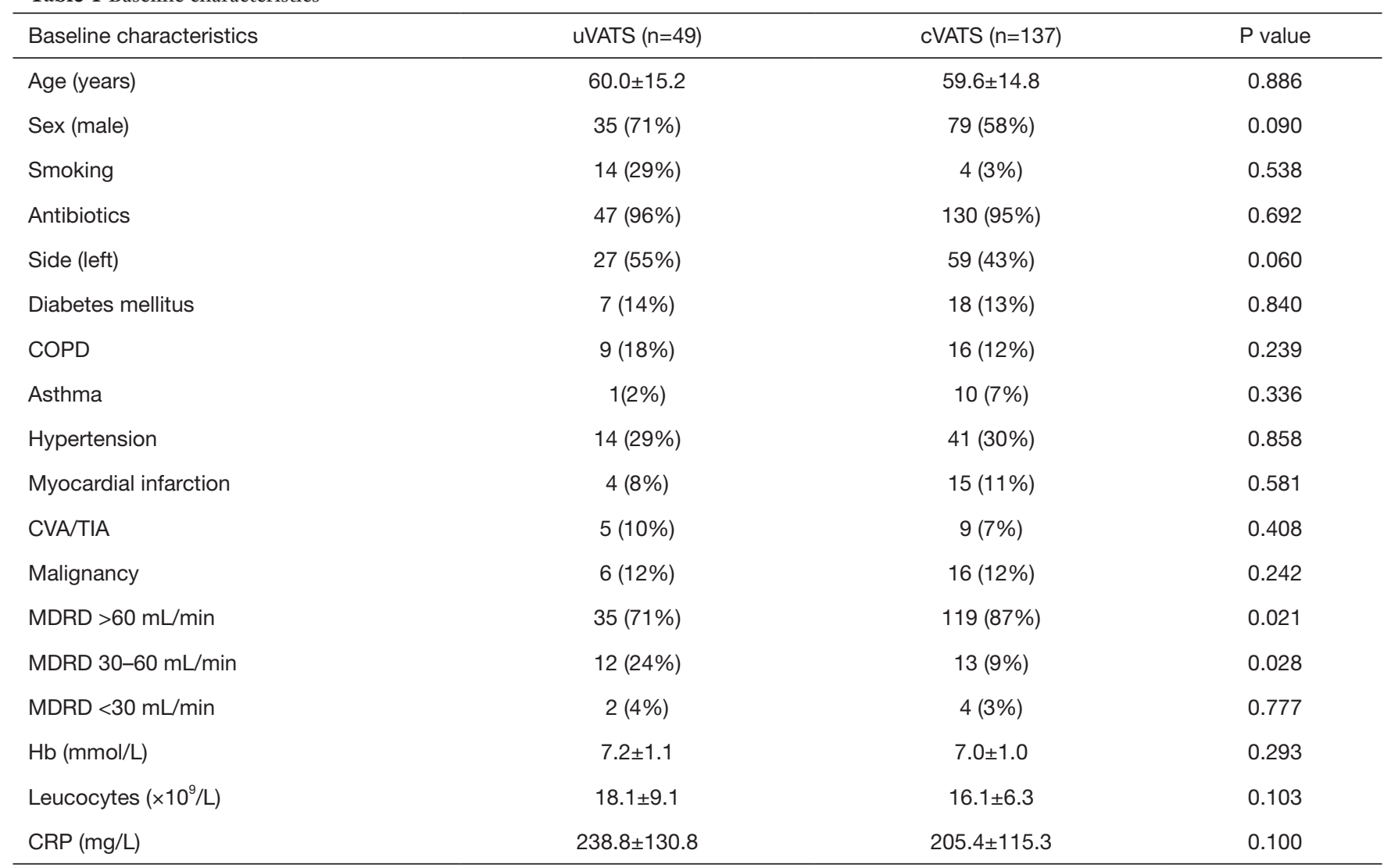

UVATS, uniportal video assisted thoracoscopy; cVATS, complete video assisted thoracoscopy; COPD, chronic obstructive pulmonary disease; CVA/TIA, cerebrovascular accident/transient ischemic attack.

compared with Chi-square-test. Continuous variables were compared with independent $t$-test. Values of $\mathrm{P}<0.05$ were considered significant.

\section{Results}

In the period studied, 186 patients were surgically treated for empyema. Of these patients, 137 were treated with cVATS and 49 were approached via uVATS. Baseline characteristics are depicted in Table 1. Except for a slightly reduced kidney function in the uVATS group $(57.3 \pm 6.3$ vs. $\left.71.4 \pm 17.2 \mathrm{~mL} / \mathrm{min} / 1.73 \mathrm{~m}^{2}, \mathrm{P} \leq 0.001\right)$, no significant differences were noted.

\section{Peropartive results}

The peropartive characteristics are listed in Table 2. The duration of uVATS was comparable to a cVATS procedure and number of peropartive complications was equal.
In the uVATS group, no conversion towards cVATS or thoracotomy was performed. In the cVATS group a low number of procedures were converted towards a posterolateral thoracotomy. In the uVATS group, more decortications were performed. Furthermore, in the cVATS group two chest tubes were placed, while in the uVATS group usually only one chest tube was used.

\section{Postoperative results}

Patients had a mean hospital stay of between two and three weeks (Table 3, no difference between groups). Duration of chest tube drainage was also similar in both groups. Complications ranged from a local wound infection towards respiratory insufficiency and even severe sepsis. Numbers are small and were not statistically different between groups. In both groups, re-interventions because of persisting empyema were low and not significantly different. Postoperative laboratory results were more or less 
Table 2 Peropartive characteristics

\begin{tabular}{lccc}
\hline Peropartive data & uVATS $(\mathrm{n}=49)$ & cVATS $(\mathrm{n}=137)$ & P value \\
\hline Conversion & $0(0 \%)$ & $4(3 \%)$ & 0.396 \\
Duration $(\mathrm{min})$ & $65.3 \pm 17.9$ & $56.4 \pm 23.2$ & 0.290 \\
Complications & $2(4 \%)$ & $12(9 \%)$ & 0.287 \\
Decortication & $22(45 \%)$ & $24(18 \%)$ & $<0.001$ \\
Drains & $1.1 \pm 0.3$ & $2.0 \pm 0.3$ & $<0.001$ \\
\hline
\end{tabular}

uVATS, uniportal video assisted thoracoscopy; cVATS, complete video assisted thoracoscopy.

Table 3 Postoperative characteristics

\begin{tabular}{lccc}
\hline Postoperative data & uVATS $(\mathrm{n}=49)$ & cVATS $(\mathrm{n}=137)$ & P value \\
\hline Hospital stay (days) & $18.9 \pm 12.8$ & $20.1 \pm 14.7$ & 0.618 \\
Chest tube (days) & $6.4 \pm 4.3$ & $8.9 \pm 6.2$ & 0.016 \\
Complications & $9(18 \%)$ & $14(10 \%)$ & 0.068 \\
Death & $4(8 \%)$ & $8(6 \%)$ & 0.530 \\
Re-operation & $2(4 \%)$ & $11(8 \%)$ & 0.368 \\
\hline
\end{tabular}

uVATS, uniportal video assisted thoracoscopy; cVATS, complete video assisted thoracoscopy.

Table 4 Postoperative laboratory results

\begin{tabular}{|c|c|c|c|c|c|c|c|c|c|}
\hline $\begin{array}{l}\text { Laboratory } \\
\text { results }\end{array}$ & \multicolumn{3}{|c|}{ Day 1} & \multicolumn{3}{|c|}{ Day 3} & \multicolumn{3}{|c|}{ Day 5} \\
\hline $\mathrm{Hb}(\mathrm{mmol} / \mathrm{L})$ & $6.4 \pm 0.9$ & $6.4 \pm 1.4$ & 0.947 & $6.3 \pm 1.0$ & $6.3 \pm 0.8$ & 0.549 & $6.4 \pm 1.0$ & $6.6 \pm 0.9$ & 0.232 \\
\hline $\begin{array}{l}\text { Leucocytes } \\
\left(\times 10^{9} / \mathrm{L}\right)\end{array}$ & $17.7 \pm 8.8$ & $15.2 \pm 5.6$ & 0.053 & $13.7 \pm 5.7$ & $13.1 \pm 6.1$ & 0.656 & $12.1 \pm 6.3$ & $10.5 \pm 4.0$ & 0.063 \\
\hline
\end{tabular}

uVATS, uniportal video assisted thoracoscopy; cVATS, complete video assisted thoracoscopy.

equal between the groups, with high inflammatory values directly postoperative and a steady decline towards day 5 postoperative (Table 4).

Death of any cause was slightly higher in the uVATS group although not significantly different $(8 \% ; n=4 v s$. $6 \% ; n=8)$. Median ASA score was 3 in both groups. There were two major causes of death in both groups, namely uncontrolled infection and cardiac related mortality. In the cVATS group five patients died of an ongoing infection, while two died because of a cardiac event. One patient died due to multi-organ failure. In the uVATS group two patients died of a cardiac event and one patient due to ongoing infection. One patient in the uVATS group was diagnosed postoperatively with stage IV lung carcinoma and wished no further treatment.

\section{Discussion}

The goal of treatment for pleural empyema is infection control, prevention of persistent or recurrent disease, and maintenance or restoration of pulmonary function. Incomplete drainage of the pleural space by chest tube in stage I empyema with signs of ongoing infection warrants surgical intervention. However, pre-operative staging of empyema can be difficult and this complicates timing of surgical procedures (15). Traditionally, these procedures mandated a thoracotomy. VATS added a minimally invasive alternative to the traditional approach. Early in the VATS 
era a complex pleural space was considered a contraindication, but as experience grew, cVATS proved to be as good as the classic approach and is adequate even in the most inflamed pleural spaces $(7,8,10,11)$. Despite the advantages, the use of cVATS is still debated in literature. A recent expert consensus of the European academy of cardiothoracic surgery stated that cVATS should be the primary approach, but that decortication can be technically demanding and a high rate of conversion to thoracotomy can be expected. Furthermore, they stated that in wellorganized empyema (symptoms $>5$ weeks) a primary thoracotomy should be considered (16). In this current study we show that cVATS is technically feasible with a low complication rate of $10 \%$. Only a small number of patients required a re-operation, mainly due to persistent empyema. These numbers are comparable to those found in literature $(7,17,18)$. In only four patients a conversion to a thoracotomy was necessary due to technical reasons. This shows that with the growing experience with cVATS almost all empyema's can be treated with a minimal invasive approach.

In the quest for further minimization of surgical trauma, uVATS is the latest evolution. By limiting surgical trauma to only one intercostal space, it aims to reduce postoperative pain and enhance recovery $(13,14)$. Although uVATS has been increasingly adapted for the management of recurrent uncomplicated pleural effusions, preoperative staging in lung cancer, treatment of primitive spontaneous pneumothorax, palmar hyperhidrosis and even for complex anatomic lung resections $(19,20)$, surprisingly little is published about its use in empyema. An early publication by Song et al., reported that a uniportal approach in empyema was difficult and resulted in a $50 \%$ conversion rate to cVATS or thoracotomy (21). Subsequent studies showed lower conversion rates of around 10\% (22).

A recent retrospective study by Ismail et al. included 35 patients from two centres. Postoperative results were good, with only minor complications in $17 \%$ of the patients (23). Furthermore, there was no conversion towards multiportal VATS or thoracotomy. Even though they removed the thickened visceral pleura to ensure to fully expand the lung. This data is comparable to our results, with no conversion necessary and low postoperative complications. The postoperative complication rate of $16 \%$ is comparable to outcomes reported in patients with empyema treated with the multiportal approach. Despite numbers are small, peri-operative mortality was higher in the uVATS than in the cVATS group $(8 \% ; \mathrm{n}=4$ vs. $6 \% ; \mathrm{n}=8)$. The difference between groups can partially be explained by the one abstinated patient with a stage IV lung carcinoma in the uVATS group. This death cannot be related to a surgical technique or ongoing infection. Furthermore, in almost half of the patients a decortication was performed to fully expand the lung, without the need for reintervention, showing that it is technically feasible to treat even the more complex pleural cavities via a uniportal technique.

uVATS is a novel technique that requires additional skills and training. Our experience is that the learning curve can be steep for surgical teams that are already experienced in cVATS. This current database represents the transition from cVATS [2006-2015] to uVATS [2016-current] as a standard surgical technique in our hospital. This may explain the slightly longer surgical time in the uVATS group. After the learning curve, we expect that the duration of uVATS will soon be comparable to cVATS.

In the cVATS group, at baseline, more patients had pathologically proven malignancies. With that in mind, the higher rate of decortication in the uVATS group is peculiar. It might be explained by better registration of that part of the procedure, or by a higher percentage of stage III empyema in the uVATS group. Unfortunately, in this retrospective database, peropartive judgement of the stages of empyema was not well documented. It illustrates however that decortication is feasible during a uniportal approach, without the need for further re-interventions or conversion.

In the cVATS group two chest tubes were placed, while in the uVATS group only one chest tube was used. The application of one or more chest tubes after pulmonary surgery appears highly various between practices (24). A motive for placement of multiple chest tubes is the assumption of more adequate drainage and the option of draining the whole pleural cavity. However, when all pockets are cleared surgically, one communicating space remains for which one chest tube should in theory be sufficient to abduct pleural fluid. The transition to the placement of only one tube is the result of minimal invasive character of the uVATS approach in which the chest tube is placed via the single incision working port. From our data it appears that insertion of only one chest tube has similar good clinical outcomes as multiple tubes.

Postoperative hospital stay and chest tube drainage was relatively long in relation to that found in literature (range, 7-16 days for hospital stay) (6). A possible explanation is the wide variation in postoperative care for patients with empyema between practices. In some hospitals, patients stay on the surgical ward until they are fit enough to go 
home, whereas in other hospitals rehabilitation is taken over by the pulmonology department at discharge from the surgical department. Due to this variation, the differences in hospital stay between publications from different centres should be interpreted with caution.

The possible drawback of minimal invasive surgery for empyema could be that less thorough evacuation of infectious fibrinopurulent discharge can be performed. Theoretically, this should result in more re-interventions. However, that is contradicted by our current data and data from others in literature $(6,7)$, demonstrating that surgical treatment for empyema with cVATS and even with uVATS is appropriate.

\section{Conclusions}

Uniportal VATS is a feasible technique in all patients with pleural empyema requiring surgery. Even if decortication in stage III empyema is required this can be performed by uniportal VATS, without the need for re-interventions. Larger series are required to define the place of uniportal VATS in the treatment of parapneumonic empyemas.

\section{Acknowledgments}

Funding: None.

\section{Footnote}

Conflicts of Interest: All authors have completed the ICMJE uniform disclosure form (available at http://dx.doi. org/10.21037/jtd.2020.02.29). The authors have no conflicts of interest to declare.

Ethical Statement: The authors are accountable for all aspects of the work in ensuring that questions related to the accuracy or integrity of any part of the work are appropriately investigated and resolved. Approval was obtained from the local ethical committee, and informed consent was waived due to the retrospective nature of this study.

Open Access Statement: This is an Open Access article distributed in accordance with the Creative Commons Attribution-NonCommercial-NoDerivs 4.0 International License (CC BY-NC-ND 4.0), which permits the noncommercial replication and distribution of the article with the strict proviso that no changes or edits are made and the original work is properly cited (including links to both the formal publication through the relevant DOI and the license). See: https://creativecommons.org/licenses/by-nc-nd/4.0/.

\section{References}

1. Andrews NC, Parker EF, Shaw RP, et al. Management of non-tuberculous empyema. Am Rev Respir Dis 1962;85:935-6.

2. Davies CW, Gleeson FV, Davies RJ, et al. BTS guidelines for the management of pleural infection. Thorax 2003;58 Suppl 2:ii18-28.

3. Yang $W$, Zhang B, Zhang ZM. Infectious pleural effusion status and treatment progress. J Thorac Dis 2017;9:4690-9.

4. Colice GL, Curtis A, Deslauriers J, et al. Medical and surgical treatment of parapneumonic effusions : an evidence-based guideline. Chest 2000;118:1158-71.

5. Farjah F, Symons RG, Krishnadasan B, et al. Management of pleural space infections: a population-based analysis. J Thorac Cardiovasc Surg 2007;133:346-51.

6. Cassina PC, Hauser M, Hillejan L, et al. Video-assisted thoracoscopy in the treatment of pleural empyema: stagebased management and outcome. J Thorac Cardiovasc Surg 1999;117:234-8.

7. Wurnig PN, Wittmer V, Pridun NS, et al. Video-assisted thoracic surgery for pleural empyema. Ann Thorac Surg 2006;81:309-13.

8. Chambers A, Routledge T, Dunning J, et al. Is videoassisted thoracoscopic surgical decortication superior to open surgery in the management of adults with primary empyema? Interact Cardiovasc Thorac Surg 2010;11:171-7.

9. Angelillo Mackinlay TA, Lyons GA, Chimondeguy DJ, et al. VATS debridement versus thoracotomy in the treatment of loculated postpneumonia empyema. Ann Thorac Surg 1996;61:1626-30.

10. Scott WJ, Allen MS, Darling G, et al. Video-assisted thoracic surgery versus open lobectomy for lung cancer: a secondary analysis of data from the American College of Surgeons Oncology Group Z0030 randomized clinical trial. J Thorac Cardiovasc Surg 2010;139:976-81; discussion 981-3.

11. Paul S, Altorki NK, Sheng S, et al. Thoracoscopic lobectomy is associated with lower morbidity than open lobectomy: a propensity-matched analysis from the STS database. J Thorac Cardiovasc Surg 2010;139:366-78.

12. Gonzalez D, Paradela M, Garcia J, et al. Single-port videoassisted thoracoscopic lobectomy. Interact Cardiovasc 
Thorac Surg 2011;12:514-5.

13. Shen Y, Wang H, Feng M, et al. Single- versus multipleport thoracoscopic lobectomy for lung cancer: a propensity-matched studydagger. Eur J Cardiothorac Surg 2016;49 Suppl 1:i48-53.

14. Akter F, Routledge T, Toufektzian L, et al. In minor and major thoracic procedures is uniport superior to multiport video-assisted thoracoscopic surgery? Interact Cardiovasc Thorac Surg 2015;20:550-5.

15. Cameron RJ. Management of complicated parapneumonic effusions and thoracic empyema. Intern Med J 2002;32:408-14.

16. Scarci M, Abah U, Solli P, et al. EACTS expert consensus statement for surgical management of pleural empyema. Eur J Cardiothorac Surg 2015;48:642-53.

17. Chan DT, Sihoe AD, Chan S, et al. Surgical treatment for empyema thoracis: is video-assisted thoracic surgery "better" than thoracotomy? Ann Thorac Surg 2007;84:225-31.

18. Lardinois D, Gock M, Pezzetta E, et al. Delayed referral and gram-negative organisms increase the conversion

Cite this article as: van Middendorp LB, Franssen S, Gillissen S, Maessen JG, Hulsewé KWE, Vissers YLJ, de Loos ER. Uniportal video-assisted thoracoscopy is a safe approach in patients with empyema requiring surgery. $\mathrm{J}$ Thorac Dis 2020;12(4):1460-1466. doi: 10.21037/jtd.2020.02.29 thoracotomy rate in patients undergoing video-assisted thoracoscopic surgery for empyema. Ann Thorac Surg 2005;79:1851-6.

19. Gonzalez-Rivas D, Sihoe ADL. Important Technical Details During Uniportal Video-Assisted Thoracoscopic Major Resections. Thorac Surg Clin 2017;27:357-72.

20. Gonzalez-Rivas D, Fernandez R, Fieira E, et al. Uniportal video-assisted thoracoscopic bronchial sleeve lobectomy: first report. J Thorac Cardiovasc Surg 2013;145:1676-7.

21. Song IH, Yum S, Choi W, et al. Clinical application of single incision thoracoscopic surgery: early experience of 264 cases. J Cardiothorac Surg 2014;9:44.

22. Bongiolatti S, Voltolini L, Borgianni S, et al. Uniportal thoracoscopic decortication for pleural empyema and the role of ultrasonographic preoperative staging. Interact Cardiovasc Thorac Surg 2017;24:560-6.

23. Ismail M, Nachira D, Meacci E, et al. Uniportal videoassisted thoracic surgery in the treatment of pleural empyema. J Thorac Dis 2018;10:S3696-703.

24. Zardo P, Busk H, Kutschka I. Chest tube management: state of the art. Curr Opin Anaesthesiol 2015;28:45-9. 